\title{
Predicting Outcome in Skull Base Osteomyelitis: An Assessment of Demographic, Clinical, and Pathological Attributes
}

\author{
Alisha Saxena ${ }^{1}$ Birinder Singh Paul ${ }^{1}$ Gagandeep Singh ${ }^{1}$ Archana Ahluwalia ${ }^{2}$ Gunchan Paul ${ }^{3}$
}

${ }^{1}$ Department of Neurology, Dayanand Medical College \& Hospital, Ludhiana, Punjab, India

2 Department of Radiodiagnosis, Dayanand Medical College \&

Address for correspondence Birinder Singh Paul, DM, Department of Neurology, Dayanand Medical College \& Hospital, Ludhiana, Punjab Hospital, Ludhiana, Punjab, India

${ }^{3}$ Department of Critical Care Medicine, Dayanand Medical College \& Hospital, Ludhiana, Punjab, India

J Neurosci Rural Pract 2021;12:751-757.

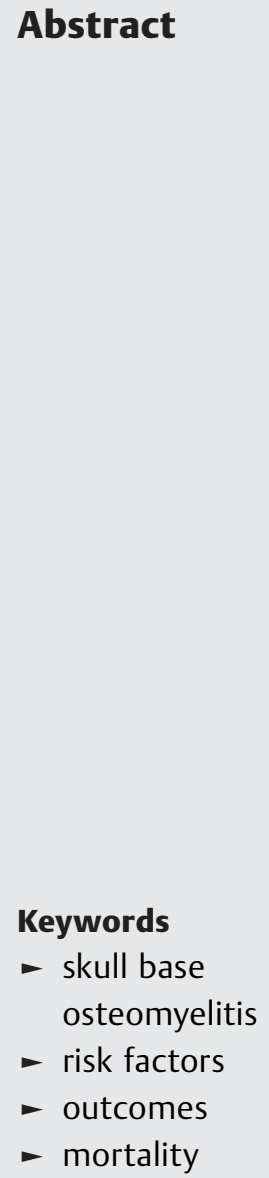

Objective Skull base osteomyelitis (SBO) is an enigmatic clinical diagnosis which is difficult to decipher and is associated with poor outcomes. The study aims to examine the demographic and clinical characteristics of patients with SBO and its outcomes. Materials and Methods Medical records of 30 patients with diagnosis of SBO over past 5 years were assessed for demographic and clinical characteristics, type of SBO, radiological parameters, treatment received, procedure performed, microbiological profile, comorbidities, and complications including cranial nerve $(\mathrm{CN})$ palsies. These factors were analyzed for prediction of outcome (death or survival).

Statistical Analysis Microsoft Office Excel 2010 SAS 10.0 for Windows was used. Student's t-test for continuous variables (age, duration of symptoms, number of days of hospitalization, and treatment duration) and chi-square test for categorical variables (imaging findings, symptomatology, presence of comorbidities, surgical procedure, complications, and type of antibiotics) were utilized.

Results We found SBO was the disease of elderly population ( $64.07 \pm 6.13$ years) with male predominance (83.3\%) highly associated with uncontrolled diabetes status (93.3\%). Headache (100\%) and CN palsy (80\%) were the most common neurological presenting complaints followed by stroke (17\%) and encephalopathy $(10 \%)$. Pathological and radiological correlation showed that fungal infection (Aspergillus) was associated with anterior SBO (10\%), while bacteria (Pseudomonas) was cultured from posterior SBO (30\%). Fifty per cent of patients were alive after 1 year out of which $33 \%$ had good functional outcome. The mortality rate was $33.3 \%$ in our cohort and multiple lower $\mathrm{CN}$ palsies $(p=0.04)$, suboptimal duration of medical treatment $(p=0.03)$, surgical intervention during clinical course $(p=0.02)$, and development of intracranial or extracranial complications $(p=0.03)$ were the predictors of mortality. Conclusion Early diagnosis including identification of pathogenic organisms and optimal duration of treatment are crucial factors for improved outcomes in SBO. published online September 28, 2021
DOI https://doi.org/ $10.1055 / \mathrm{s}-0041-1735324$ ISSN 0976-3147. (c) 2021. Association for Helping Neurosurgical Sick People. All rights reserved.

This is an open access article published by Thieme under the terms of the Creative Commons Attribution-NonDerivative-NonCommercial-License, permitting copying and reproduction so long as the original work is given appropriate credit. Contents may not be used for commercial purposes, or adapted, remixed, transformed or built upon. (https://creativecommons.org/ licenses/by-nc-nd/4.0/)

Thieme Medical and Scientific Publishers Pvt. Ltd., A-12, 2nd Floor, Sector 2, Noida-201301 UP, India 


\section{Introduction}

Skull base osteomyelitis (SBO) is inflammation of the base of calvarium caused by microbial organisms. ${ }^{1}$ The skull base is composed of frontal, ethmoid, sphenoid, parietal, temporal, and occipital bones from anterior to posterior. It is divided by sphenoidal ridge anteriorly and petrous ridge posteriorly into three cranial fossae-anterior, middle, and posterior. ${ }^{2}$ The diagnosis of SBO can be made on radiological findings but requires a high degree of clinical suspicion. SBO still remains a diagnostic and therapeutic challenge as many clinicians are unaware of this condition and its long-term prognosis. Despite advancement in diagnostic and treatment modalities, the reported mortality is as high as $53 \%{ }^{3}$ The first review of chronic SBO was published in 1961 in only three patients, and highlights the rarity of the condition and difficulty in localizing the lesion. ${ }^{4} \mathrm{~A}$ retrospective analysis of five patients has emphasized the importance of prompt diagnosis and optimal duration of antibiotics in SBO. ${ }^{5}$

We report clinical manifestations, diagnostic characteristics, and functional outcomes in a series of 30 patients of SBO from a single tertiary care referral hospital. This is the first study to assess the functional outcomes and long-term survival in patients of SBO using standard neurological scales.

\section{Materials and Methods}

\section{Study Population}

We conducted a retrospective cohort study of 30 consecutive subjects who were admitted in the departments of neurology, neurosurgery, otolaryngology, and internal medicine in Dayanand Medical College and Hospital, Ludhiana, Punjab over a 5-year period (2015-2020). The study was undertaken with the approval from the local Institutional Ethics Committee (2020-544).

\section{Case Definitions}

The diagnosis of SBO was considered in patients who had osteomyelitis of clivus, petrous bone, or any part of base of skull confirmed on contrast-enhanced magnetic resonance imaging (MRI) of the brain or computed tomography (CT) of the head in addition to history of headache and cranial nerve $(\mathrm{CN})$ palsies. Typical SBO or malignant otitis externa included osteomyelitis secondary to temporal bone infection, whereas when SBO was caused without any such preceding infection, it was classified as atypical. ${ }^{6}$ Anterior, central, and posterior SBOs were categorized according to skull base anatomy-anterior clinoid process and lesser wing of sphenoid constitute anterior, greater wing of sphenoid and petrous part of temporal bone denote central, and clivus involvement is indicative of posterior $\mathrm{SBO} .^{7} \mathrm{CN}$ palsy was classified as single or multiple and in case of multiple, the patients were further categorized as predominantly upper CN palsy (I-VI) or lower CN palsy (VII-XII) or both. All patients with postoperative involvement of temporal bone flap and posttraumatic or iatrogenic osteomyelitis of skull base were excluded.

\section{Parameters Assessed}

The following information was extracted through patients' chart review and entered into a structured pro forma: clinical and demographic characteristics, type of SBO based on skull bones involved, and radiological data. Detailed neurological examination including $\mathrm{CN}$ palsies and other neurological deficits was also noted from patients' records. Any history of ear discharge or nasal symptoms, laboratory data including complete hemogram, blood cultures, erythrocyte sedimentation rate, and contrast-enhanced MRI of the brain and CT of the head were recorded. Hospital course including duration of stay, treatment received (antibiotics/antifungals, dosage, and duration), any diagnostic or therapeutic surgical interventions, and presence of any complication related to the disease developed during their hospital course were entered into the pro forma.

\section{Outcomes Assessed}

Outcome of all patients with the diagnosis of SBO was dichotomized as poor outcome (death) or survival. This was done by telephonic interview in 25 out of 30 patients as 5 could not be contacted and were lost to follow-up.

Standardized consent for analyzing and publishing the data was taken during the telephonic interview. In the patients with SBO who had died, we used the verbal autopsy questionnaire (shortened PHMRC [Population Health Metrics Research Consortium] verbal autopsy instrument) to ascertain whether the cause of death was related to SBO or to their previous comorbid conditions. ${ }^{8}$ Time duration between discharge and death was noted. For the survivors, functional outcome was assessed at 6 and 12 months with modified Rankin scale (mRS) and Glasgow outcome scale (GOS). ${ }^{9,10}$

\section{Statistical Analysis}

Statistical analysis was done with the help of Microsoft Office Excel 2010 SAS 10.0 for Windows. The age (years) of subjects, duration of symptoms, hospital stay, and treatment were noted as continuous variables. The presence of any comorbid condition, symptomatology, imaging findings, any procedure done, complications during hospital course, and type of antibiotics were considered as categorical variables.

Continuous variables were demonstrated as mean \pm standard deviation. Student's $t$-test for continuous variables and chi-square test for categorical variables were used to chart the comparisons of these variables between those who died or survived.

\section{Results}

\section{Demography}

In this cohort of 30 patients, 25 (83.3\%) patients were males with mean age of $64.07 \pm 6.13$ years. Diabetes mellitus was the most common comorbid condition (93.3\%) with others being hypertension, chronic kidney disease, chronic liver disease, and coronary artery disease. Baseline and demographic characteristics of the cohort are described in - Table $\mathbf{1}$. 
Table 1 Baseline characteristics of 30 patients in the study

\begin{tabular}{|c|c|}
\hline Baseline characteristics & $\begin{array}{l}\text { Number of } \\
\text { patients (\%) } \\
N=30\end{array}$ \\
\hline \multicolumn{2}{|l|}{ Gender } \\
\hline Male & $25(83.3)$ \\
\hline Female & 05 (16.6) \\
\hline Mean age $(y)$ & $64.07 \pm 6.13(46-81)$ \\
\hline \multicolumn{2}{|l|}{ Side of SBO } \\
\hline Right & $14(46.6)$ \\
\hline Left & $14(46.6)$ \\
\hline Bilateral & $02(6.6)$ \\
\hline \multicolumn{2}{|l|}{ Clinical complaints } \\
\hline Headache & $30(100)$ \\
\hline \multicolumn{2}{|l|}{ Neurological examination } \\
\hline \multicolumn{2}{|l|}{ Cranial nerve palsies } \\
\hline Upper (I-VI) & $02(6.6)$ \\
\hline Lower (VII-XII) & $24(80)$ \\
\hline Both set of cranial nerves & $04(13.3)$ \\
\hline \multicolumn{2}{|l|}{ Comorbidities } \\
\hline Diabetes mellitus & $28(93.3)$ \\
\hline Hypertension & $18(60)$ \\
\hline CKD & $06(20)$ \\
\hline CLD & $03(10)$ \\
\hline CAD & $03(10)$ \\
\hline Systemic vasculitis & $02(6.6)$ \\
\hline \multicolumn{2}{|l|}{ Intracranial complications } \\
\hline Stroke & $11(36.6)$ \\
\hline Anterior circulation infarct & $04(13.3)$ \\
\hline Brain stem infarct & $01(3.3)$ \\
\hline Bezold's abscess & $01(3.3)$ \\
\hline Encephalopathy & $03(10)$ \\
\hline $\begin{array}{l}\text { Temporomandibular } \\
\text { joint involvement }\end{array}$ & $02(6.6)$ \\
\hline ESR & $78.26 \pm 27.71(09-132)$ \\
\hline \multicolumn{2}{|l|}{ Procedure } \\
\hline Tympanomastoid procedure & $12(40)$ \\
\hline Mastoidectomy & $09(30)$ \\
\hline Diagnostic biopsy (FESS) & $03(10)$ \\
\hline \multicolumn{2}{|l|}{ Radiological profile } \\
\hline Atypical & $24(80)$ \\
\hline Typical & $06(20)$ \\
\hline Anterior & $03(10)$ \\
\hline Posterior & $09(30)$ \\
\hline
\end{tabular}

Abbreviations: CAD, coronary artery disease; CKD, chronic kidney disease; CLD, chronic liver disease; ESR, erythrocyte sedimentation rate; FESS, functional endoscopic sinus surgery; SBO, skull base osteomyelitis.

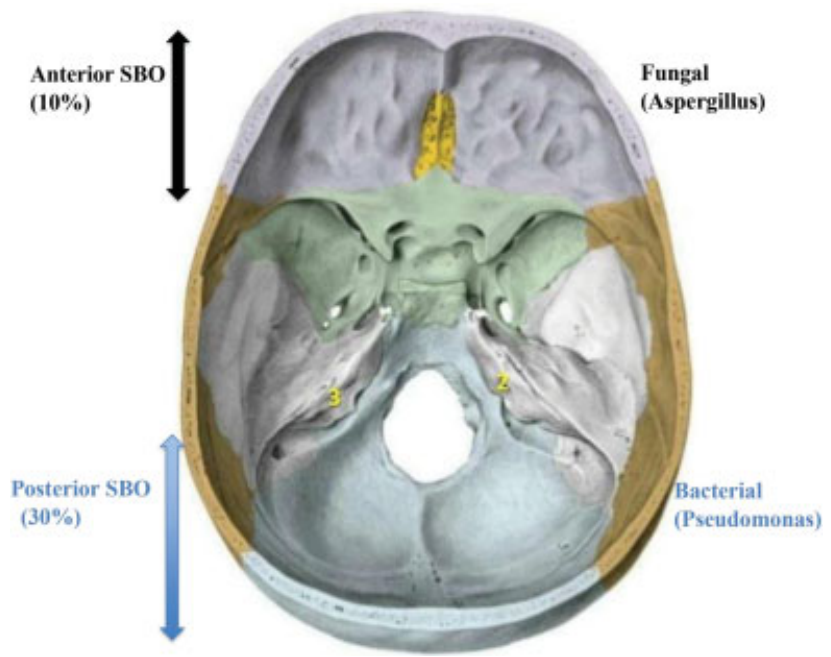

Fig. 1 Classification of skull base osteomyelitis (SBO) along with causal microorganisms.

\section{Clinical Characteristics}

Headache was the presenting symptom in 30 patients (100\%). All patients with SBO had CN palsies either single or multiple. Ten (33.3\%) patients had one $\mathrm{CN}$ involvement, while 20 (66.6\%) had more than one $\mathrm{CN}$ involvements. Lower CNs (VII-XII) were more commonly involved (80\%).

\section{Imaging Findings}

According to MRI, 18 (60\%) patients had central (atypical), 9 (30\%) had radiological findings suggestive of posterior, while 3 (10\%) patients had anterior SBO (-Fig. 1). Single photon emission computed tomography/CT was done on one patient and it revealed three foci of osseous overactivity in mastoid process of left temporal bone with extension into middle ear, petrous apex, and left temporomandibular (TM) joint (- Fig. 2).

\section{Hospital Course}

\section{Procedure}

Only 12 patients underwent surgical intervention. Cultures revealed growth of Pseudomonas in nine (30\%) patients, while two out of three (10\%) patients who had radiological findings of anterior SBO had fungal growth in their diagnostic endoscopy biopsy.

\section{Complications}

In our study, 11 (36.6\%) patients suffered additional intracranial complications, 4 had anterior circulation stroke, while 1 had posterior circulation stroke. Encephalopathy was observed in one patient. One patient developed Bezold's abscess, while right TM joint osteomyelitis was noted in two patients.

\section{Treatment Regimens}

All patients were treated with intravenous broad-spectrum antimicrobials to cover entire range of putative microorganisms during their hospital stay which included piperacillintazobactam in 18 (60\%), carbapenems in 6 (20\%), 


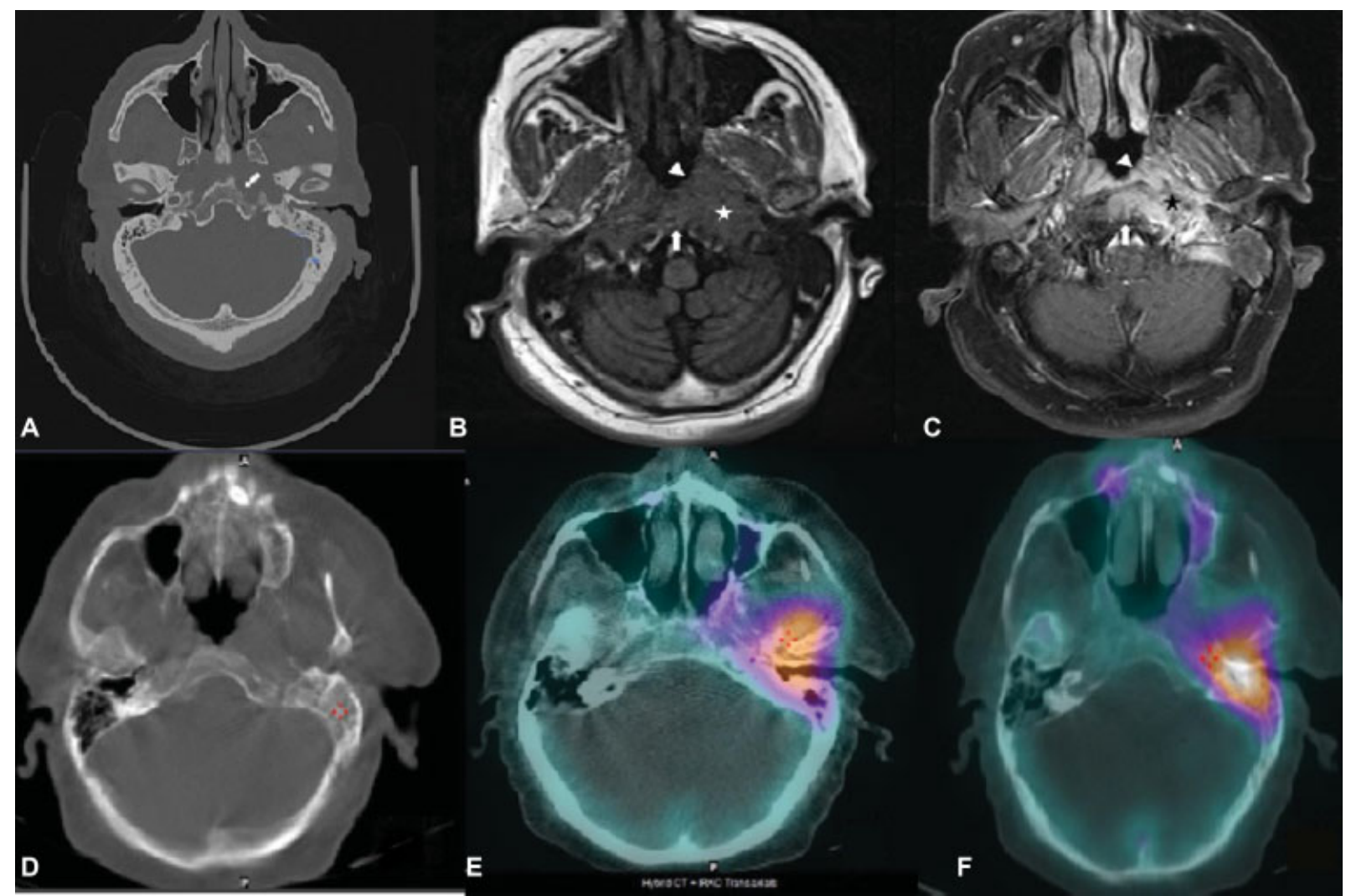

Fig. 2 First row image: (A) Axial computed tomography (CT) demonstrates erosion of cortex along left lateral clivus and left petrous apex (arrow), (B) axial T1-weighted magnetic resonance imaging, and (C) magnetic resonance postcontrast T1 show infiltrative soft tissue in submucosa of nasopharynx on left side (arrowhead) extending posteriorly to paraclival tissue and left carotid space (star); in addition, marrow of clivus and basiocciput was involved by infective process (arrow). Second row image: (D) Bone CT shows destruction of left mastoid, (E) bone single photon emission computed tomography showing high-grade uptake in left petrous apex, and (F) left temporomandibular joint.

cephalosporins in 5 (16.6\%), and vancomycin in 1 (3.3\%) patient. They were advised to continue oral antimicrobials following discharge; however, only half of patients took treatment for sufficient time period (6-20 weeks). ${ }^{11}$ The mean duration of treatment was 71 days (range: 4-210 days). Patients were categorized as having received adequate treatment if they fulfilled the parameters of treatment as given in - Fig. 3 .

\section{Outcomes}

Outcome assessment was possible in 25 patients as 5 were lost to follow-up. The mortality rate in our cohort was $33.3 \%$ out of which two patients died in hospital in whom the cause of death was directly related to SBO, that is, aspiration pneumonitis due to bulbar palsy, while eight patients died after discharge from hospital. After discharge, the cause of death as determined with the help of Verbal Autopsy Questionnaire was aspiration in four (55\%) and neurologically deteriorating condition in three patients and one patient who was a recipient of renal transplant died due to sepsis. The mean duration of survival among those who died after discharge was 43 days. After 1 year, 15 (50\%) patients of SBO were alive among whom 10 (33.3\%) showed good functional outcome as per the neurological scales $-\mathrm{mRS}<3$ and GOS $>1$, while 5 needed some form of assistance in their routine daily activities.

\section{Discussion}

SBO is a complex pathological condition associated with a high morbidity and mortality despite advancements in diagnostic modalities and management techniques. The diagnosis of disease is challenging because there are no set diagnostic criteria. In this study, we tried to access the knowledge gap in SBO by addressing its clinical, laboratory, and radiological characteristics and emphasize the factors affecting survival.

Poorly controlled diabetes mellitus with duration more than 5 years was the major comorbid condition associated with SBO in our cohort (93.3\%). There was high frequency of SBO in elderly in their sixth decade of life (mean age 64 years) with a male preponderance (83.3\%). Two patients, one with vasculitis and other on immunosuppressants were younger in the cohort (46 and 52 years). Headache was the most common symptom in all patients and the presenting symptom in $80 \%$. Multiple $\mathrm{CN}$ palsies was the second most common neurological finding, lower $\mathrm{CN}$ palsy being more commonly seen (80\%) than upper (6.6\%). One patient had six $\mathrm{CN}$ palsies (VII-XII). The presence of multiple lower $\mathrm{CN}$ palsies was associated with increased mortality $(p=0.04)$. In our study, we found 24 (80\%) patients had atypical SBO. The major neurological complications associated in these patients were stroke (17\%) and encephalopathy (10\%), while 


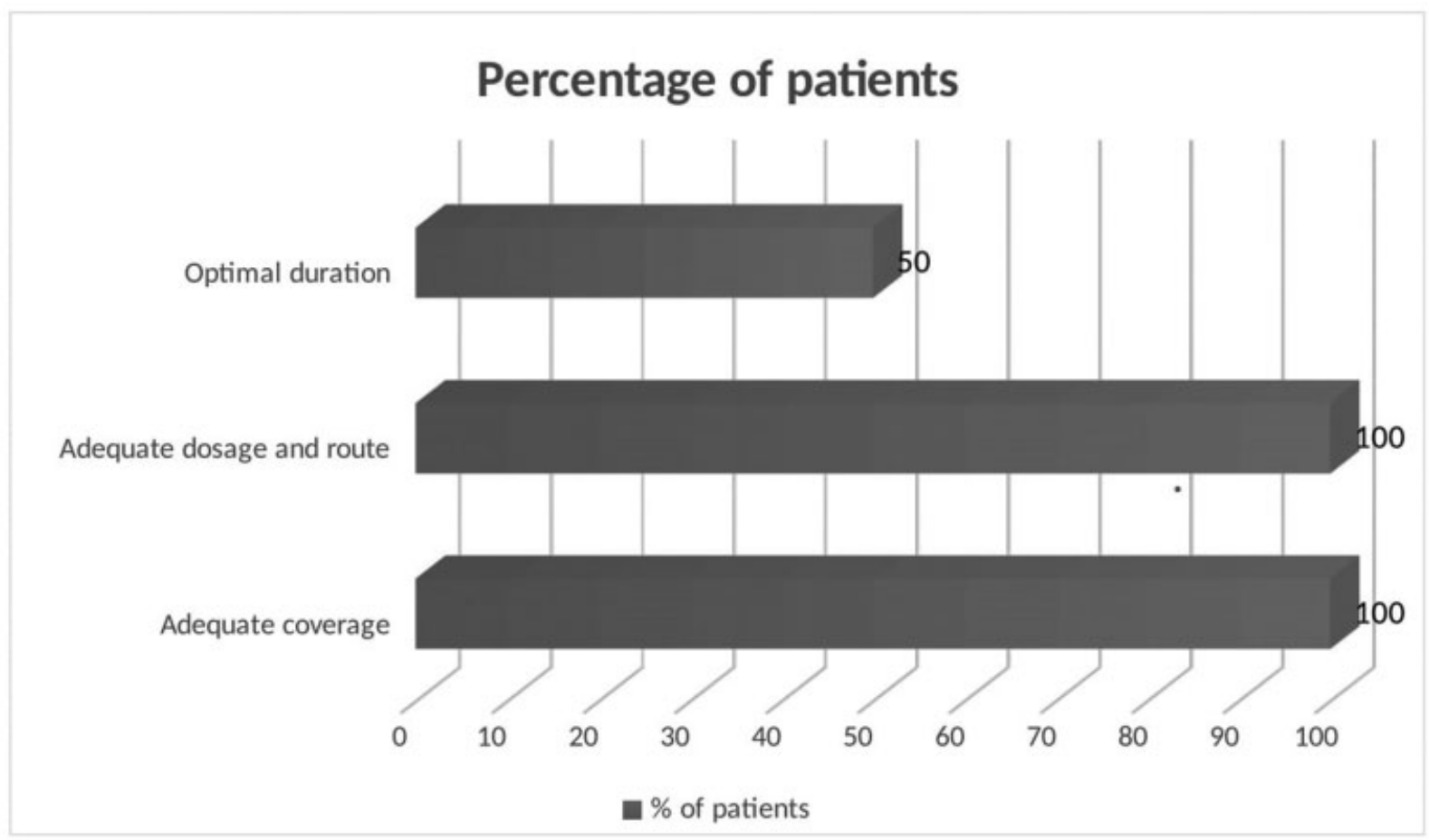

Fig. 3 Percentage of patients who received adequate treatment.

distant noncontiguous abscess (3\%) and infective arthritis of TM joint (7\%) were also observed. The presence of any of these complications was a strong predictor of mortality $(p=0.03)$ and this has not been reported previously. Diagnostic or therapeutic procedure was done in $12(40 \%)$ and pathogen was cultured in 11 (37\%) patients. Though infectious process in SBO can be caused by wide range of pathogens; however, in our study, Pseudomonas was isolated from nine (30\%), while Aspergillus from two (7\%) patients. On pathogenic and radiological correlation, Aspergillus was more commonly associated with anterior (10\%) and Pseudomonas with posterior SBO (30\%). Poor outcome was noted in patients in whom the aforementioned procedure was done $(p=0.02)$. In our cohort, $24(80 \%)$ patients were discharged on home-based care. On critically appraising the treatment, $50 \%$ received suboptimal treatment (duration $<6-20$ weeks). On further analysis, we found that inadequate treatment was the key factor for poor outcome $(p=0.03)$. After 1 year follow-up, 50\% patients with SBO were alive out of which $33 \%$ had good functional outcome ( $\mathrm{mRS}<3$ and GOS $>1$ ). Mortality rate noted in our cohort was $33 \%$.

Poorly controlled glycemic status leads to poor microcirculation, causing endarteritis and impaired immunity, also advancing age with presence of comorbidity leads to decreased bone vascularity and hence, increasing the susceptibility for infections. ${ }^{12,13}$ Headache in SBO is related to dural inflammation, bone destruction, or abscess formation. ${ }^{14}$ The presence of lower CN palsy may be related to spread of infection beyond the temporal bone into posteromedial area of skull base resulting in involvement of jugular foramen and paralysis of IX to XI CNs. Cerebrovascular accident may be related to invasion of the blood vessels by infection leading to vasculitis. ${ }^{12}$ Also, enhanced atherosclerosis due to advanced age and presence of comorbidities such as diabetes mellitus or chronic kidney disease may also be the implicating factors. Extension of infection into the infratemporal fossa and deep cervical fascia may have led to the formation of Bezold's abscess. In the current study, poor outcome was noted even after the therapeutic or diagnostic procedure as compared with those who had no procedure $(p=0.02)$. Though debridement of necrotic bony tissue was done in only $40 \%$ of patients, remaining had diagnostic procedure, still this could not prevent the disease from progressing. However, this does not suggest that surgery by itself was not useful, in fact patients who needed the procedure were already in advanced stage of the disease. Also, debridement may itself expose the healthy bone to invading pathogens causing aggressive disease progression. ${ }^{15}$

The high frequency of SBO in elderly male diabetics was also reported previously. ${ }^{16,17}$ Previous studies have reported isolated VII or VIII CN palsy which may be because of inclusion of patients with malignant otitis externa. ${ }^{18}$ On the contrary, in our cohort, majority had atypical SBO (80\%) with no obvious clinical foci of infection elsewhere. Venous thrombosis is said to be more commonly associated with SBO, but we did not encounter any such patient. ${ }^{19}$ Rather, we had patients with anterior and posterior circulation strokes and presence of any such complication was a strong predictor of mortality $(p=0.03)$. MRI and CT scans were the most important radiological modalities for diagnosis, classification of SBO, and establishing the extent of disease. Chandler et al reported four cases, while Singh et al published 10 patients with atypical SBO diagnosed on contrast-enhanced MRI showing lytic lesion involving clivus, 
Table 2 Comparison between alive and dead patients

\begin{tabular}{|c|c|c|c|c|c|c|}
\hline & & \multicolumn{2}{|l|}{ Outcome } & \multirow[t]{2}{*}{ Chi-square value } & \multirow[t]{2}{*}{$p$-Value } & \multirow[t]{2}{*}{ Odds ratio $(95 \% \mathrm{Cl})$} \\
\hline & & Alive $\boldsymbol{n}(\%)$ & Death $n(\%)$ & & & \\
\hline \multirow[t]{2}{*}{ Age group (y) } & $<60$ & $6(40)$ & $3(30)$ & \multirow[t]{2}{*}{0.260} & \multirow[t]{2}{*}{0.610} & \multirow[t]{2}{*}{$1.56(0.28-8.53)$} \\
\hline & $>60$ & $9(60)$ & $7(70)$ & & & \\
\hline \multirow[t]{2}{*}{ Gender } & Female & $2(13)$ & $3(30)$ & \multirow[t]{2}{*}{1.042} & \multirow[t]{2}{*}{0.307} & \multirow[t]{2}{*}{$0.36(0.05-2.68)$} \\
\hline & Male & $13(87)$ & $7(70)$ & & & \\
\hline \multirow[t]{2}{*}{ Cranial nerve } & Lower & $10(67)$ & $10(100)$ & \multirow[t]{2}{*}{4.167} & \multirow[t]{2}{*}{0.041} & \\
\hline & Upper & $5(33)$ & & & & \\
\hline \multirow[t]{2}{*}{ Treatment adequacy } & No & $9(60)$ & $10(100)$ & \multirow[t]{2}{*}{5.263} & \multirow[t]{2}{*}{0.031} & \\
\hline & Yes & $6(40)$ & & & & \\
\hline \multirow[t]{2}{*}{ Complication } & No & $12(80)$ & $3(30)$ & \multirow[t]{2}{*}{6.250} & \multirow[t]{2}{*}{0.034} & \multirow[t]{2}{*}{$9.33(1.47-59.48)$} \\
\hline & Yes & $3(20)$ & $7(70)$ & & & \\
\hline \multirow[t]{2}{*}{ Procedure } & No & $13(87)$ & $4(40)$ & \multirow[t]{2}{*}{6.005} & \multirow[t]{2}{*}{0.028} & \multirow[t]{2}{*}{$9.75(1.38-68.78)$} \\
\hline & Yes & $2(13)$ & $6(60)$ & & & \\
\hline
\end{tabular}

Abbreviation: $\mathrm{Cl}$, confidence interval.

Note: $p$-values in bold are statistically significant $(p<0.05)$.

sphenoid, and petrous bones in the absence of malignant otitis externa. ${ }^{3,20}$ Literature recommends aggressive treatment for minimum of 6 to 20 weeks due to poor vascularity of target area and impaired immune response associated with underlying comorbidities. ${ }^{11,12}$ Mortality rate noted in our cohort was $33 \%$ as compared with 10 to $20 \%$ in previous literature. $^{21}$

Predictors of mortality in patients with SBO were presence of multiple lower $\mathrm{CN}$ palsies $(p=0.04)$, surgical intervention during their clinical course $(p=0.02)$, suboptimal duration of medical treatment $(p=0.03)$, or those who developed intracranial or extracranial complications $(p=0.03)$ as shown in - Table 2 . The presence of these factors in combination or isolation signifies extensive infectious process involving bones of skull base, foramina, soft tissue, as well as blood vessels. In addition, if duration of antibiotics was not adequate, it becomes difficult to curtail the infection which leads to persistent disease hence increased chances of mortality.

Our study is not without limitations. The retrospective data collection of 30 patients may have introduced a recording bias. This may be because the incidence of disease is low. The follow-up of patients was done telephonically and no advanced neurosurgical procedure was performed during the hospital stay. Since our aim was to assess mortality rather than radiological cure, follow-up MRI was not done.

\section{Conclusion}

In our study, mortality was related to lower CN palsies (VIXII), presence of neurological complications, inadequate treatment, and any surgical intervention, whereas age, gender, presence of diabetes mellitus or any other comorbidity, and type of SBO (typical or atypical) did not predict mortali- ty. The optimal treatment and its duration are still a perplex issue and evidence-based studies are needed to guide the clinicians in this regard. Our attempt to study functional outcomes in patients with the help of standardized neurological scales can prove to be a useful tool for clinicians to monitor patients on treatment of SBO. The most critical factor that holds utmost importance is the clinching of diagnosis at an early stage and sustained prolonged treatment with monitoring of compliance for patients of SBO.

\section{Funding}

None.

\section{Conflict of Interest}

None declared.

\section{Acknowledgment}

The authors would like to thank their patients for participating and sharing their data for this study.

\section{References}

1 Pincus DJ, Armstrong MB, Thaller SR. Osteomyelitis of the craniofacial skeleton. Semin Plast Surg 2009;23(02):73-79

2 Raut AA, Naphade PS, Chawla A. Imaging of skull base: pictorial essay. Indian J Radiol Imaging 2012;22(04):305-316

3 Chandler JR, Grobman L, Quencer R, Serafini A. Osteomyelitis of the base of the skull. Laryngoscope 1986;96(03):245-251

4 Askenasy HM, Kosary IZ, Braham J. Chronic osteomyelitis of base of skull. BMJ 1961;1(5224):474-475

5 Muranjan SN, Khadilkar SV, Wagle SC, Jaggi ST. Central skull base osteomyelitis: diagnostic dilemmas and management issues. Indian J Otolaryngol Head Neck Surg 2016;68(02):149-156

6 Ridder GJ, Breunig C, Kaminsky J, Pfeiffer J. Central skull base osteomyelitis: new insights and implications for diagnosis and treatment. Eur Arch Otorhinolaryngol 2015;272(05):1269-1276 
7 Álvarez Jáñez F, Barriga LQ Iñigo TR, Roldán Lora F. Diagnosis of skull base osteomyelitis. Radiographics 2021;41(01):156-174

8 Serina P, Riley I, Stewart A, et al. A shortened verbal autopsy instrument for use in routine mortality surveillance systems. BMC Med 2015;13:302

9 Banks JL, Marotta CA. Outcomes validity and reliability of the modified Rankin scale: implications for stroke clinical trials: a literature review and synthesis. Stroke 2007;38(03):1091-1096

10 McMillan T, Wilson L, Ponsford J, Levin H, Teasdale G, Bond M. The Glasgow outcome scale - 40 years of application and refinement. Nat Rev Neurol 2016;12(08):477-485

11 Khan MA, Quadri SAQ Kazmi AS, et al. A comprehensive review of skull base osteomyelitis: diagnostic and therapeutic challenges among various presentations. Asian J Neurosurg 2018;13(04): 959-970

12 Chen CN, Chen YS, Yeh TH, Hsu CJ, Tseng FY. Outcomes of malignant external otitis: survival vs mortality. Acta Otolaryngol 2010;130(01):89-94

13 Sokołowski J, Lachowska M, Karchier E, Bartoszewicz R, Niemczyk K. Skull base osteomyelitis: factors implicating clinical outcome. Acta Neurol Belg 2019;119(03):431-437

14 Fontaine D, Almairac F, Santucci S, et al. Dural and pial painsensitive structures in humans: new inputs from awake craniotomies. Brain 2018;141(04):1040-1048
15 Amorosa L, Modugno GC, Pirodda A. Malignant external otitis: review and personal experience. Acta Otolaryngol Suppl 1996;521:3-16

16 Clark MP, Pretorius PM, Byren I, Milford CA. Central or atypical skull base osteomyelitis: diagnosis and treatment. Skull Base 2009;19(04):247-254

17 Lee SC, Kim JH, Kim CH, Kim BJ. Temporomandibular joint disorder from skull-base osteomyelitis: a case report. Maxillofac Plast Reconstr Surg 2015;37(01):39

18 Arsovic N, Radivojevic N, Jesic S, Babac S, Cvorovic L, Dudvarski Z. Malignant otitis externa: causes for various treatment responses. J Int Adv Otol 2020;16(01):98-103

19 Das S, Iyadurai R, Gunasekaran K, et al. Clinical characteristics and complications of skull base osteomyelitis: a 12-year study in a teaching hospital in South India. J Family Med Prim Care 2019;8 (03):834-839

20 Singh U, Venkitachalam S, Chinnusamy R. Clinical profiling and management outcome of atypical skull base osteomyelitis. $\mathrm{Br} \mathrm{J}$ Neurosurg 2020;34(06):686-689

21 Conde-Díaz C, Llenas-García J, Parra Grande M, Terol Esclapez G, Masiá M, Gutiérrez F. Severe skull base osteomyelitis caused by Pseudomonas aeruginosa with successful outcome after prolonged outpatient therapy with continuous infusion of ceftazidime and oral ciprofloxacin: a case report. J Med Case Reports 2017;11(01):48 